\title{
Interactions between energy surplus and short-term exercise on glucose and insulin responses in healthy people with induced, mild insulin insensitivity
}

\author{
Todd A. Hagobian, Barry Braun* \\ Energy Metabolism Laboratory, Department of Exercise Science, University of Massachusetts, Amherst, MA 01003, USA
}

\begin{abstract}
Short-term exercise can enhance insulin action, but the effect may be negated by the opposing action of energy surplus. The purpose of this investigation was to test the hypothesis that a single exercise bout would increase insulin action, even when opposed by a concurrent energy surplus. After 2 days in energy balance without exercise, baseline glucose and insulin areas under the curve and the insulin sensitivity index (C-ISI) were measured during an oral glucose tolerance test in 9 healthy, habitually active subjects (6 males, 3 females). A state of relative insulin insensitivity was then induced by systematic overfeeding (OF) to generate a daily energy surplus of $768 \pm 203 \mathrm{kcal} / \mathrm{d}$ for 3 days, and the oral glucose tolerance test was repeated. In the following 24 hours, the OF was increased $\sim 2$-fold $(+6284 \pm 1669 \mathrm{~kJ} / \mathrm{d})$ and subjects performed a single bout of exercise (expenditure $=3063 \pm 803 \mathrm{~kJ})$ to maintain the same energy surplus $(+3125 \pm 993 \mathrm{~kJ} / \mathrm{d}$; OF and exercise) as OF. After OF, fasting insulin tended to be higher $(+36 \%, P=.099)$, insulin AUC rose by $38 \%(P=.002)$, and C-ISI declined from $6.6 \pm 3.1$ to $4.6 \pm 1.8(P=.007)$ compared with baseline. After OF and exercise, fasting insulin remained elevated ( $+43 \%$ compared with baseline; $P=.043)$ and C-ISI rose only slightly $(4.6 \pm 1.8$ to $5.2 \pm 2.3 ; P=.058)$, but insulin AUC declined by $20 \%(P=.048)$ compared with OF. A single exercise bout, opposed by a concurrent energy surplus, decreased the insulin response to a glucose challenge, but only partially restored the insulin AUC to baseline and had no impact on C-ISI or fasting insulin concentrations.
\end{abstract}

\section{Introduction}

Physical exercise increases blood glucose uptake, and this effect persists in the form of enhanced insulin-mediated glucose uptake (insulin sensitivity) for up to several days postexercise [1-3]. Adaptations to long-term training are not required for this effect to be observed as even a single bout of exercise will increase insulin-mediated blood glucose uptake or reduce the insulin response to an oral glucose challenge $[2,4]$. However, because the exercise energy expenditure has rarely been replaced in these short-term studies, the results may be at least partially explained by the concurrent energy deficit. The few short-term studies in which exercise energy expenditure was deliberately replaced to maintain energy balance have provided mixed

\footnotetext{
* Corresponding author. Tel.: +1 413577 0146; fax: +1 4135452906. E-mail address: bbraun@excsci.umass.edu (B. Braun).
}

results, with meal composition, timing of the measurements, degree of glycogen depletion, and/or subject characteristics potentially mediating the response [5-12]. Recently, Fox et al [8] reported that the glucose and insulin responses to oral glucose were not affected by adding dietary energy to maintain energy balance when energy was added purely in the form of lipid.

Short-term energy surplus (3-7 days when dietary intake exceeds expenditure) usually causes a relative insulin resistance, even in the absence of significant weight gain [13-15]. The impact of energy surplus in modulating postexercise insulin action has rarely been studied, although the metabolic situation is interesting from a basic science perspective (what are net effects of 2 opposing "forces") and potential real-world application (eg, dietary recommendations for active individuals). Recently, Schenk et al [11] tested the independent effects of exercise and energy surplus on insulin sensitivity and reported that infusing lipid to 


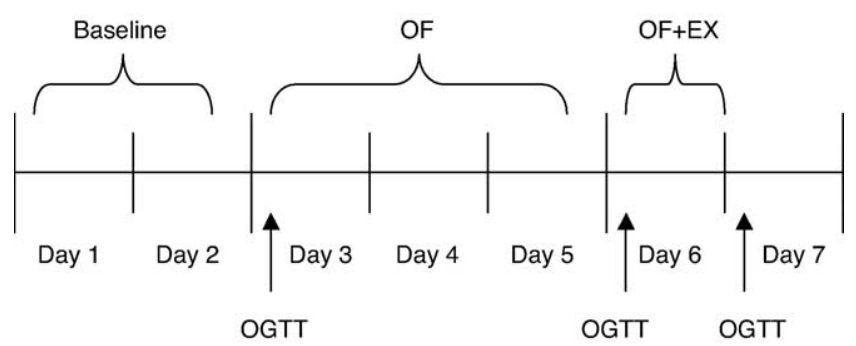

Fig. 1. Timeline of activities during the intervention.

generate an energy surplus of $1100 \mathrm{kcal}$ did not blunt insulin sensitivity after one bout of exercise in young women. In rats, however, overfeeding (OF) blunted the effects of exercise training on insulin sensitivity when compared with an energy balance condition [16,17].

To date, no studies in humans have evaluated the impact of energy surplus on postexercise insulin action with nutrients consumed rather than infused and the macronutrient composition of the diet held constant. Based on prior data $[13,15]$, we used a short-term energy surplus combined with detraining to induce a state of relative insulin insensitivity in habitually active individuals. The purpose of the current investigation was to test the hypothesis that a single bout of exercise, combined with OF to generate a considerable energy excess, would reverse the insulin insensitivity caused by the short-term energy surplus and detraining model.

\section{Methods}

\subsection{Subjects}

Six healthy males $(30 \pm 8$ years, $78.6 \pm 6.1 \mathrm{~kg}, 14.7 \% \pm$ $5.3 \%$ body fat, $60.4 \pm 6.8 \mathrm{~mL} \cdot \mathrm{kg}^{-1} \cdot \mathrm{min}^{-1}$ ) and 3 healthy females $(23 \pm 2$ years, $67.6 \pm 22.8 \mathrm{~kg}, 27.6 \% \pm$ $12.7 \%$ body fat, $44.1 \pm 7.4 \mathrm{~mL} \cdot \mathrm{kg}^{-1} \cdot \mathrm{min}^{-1}$ ) participated in the study. Data from 1 subject were dropped from the final analysis for reasons detailed below (final $\mathrm{n}=9$ ). All were nonsmokers, in excellent overall health, reported being weight stable for the previous 6 months, and participated in regular aerobic exercise (range, 5-8 h/wk). All women were studied in the follicular phase of the menstrual cycle based on the date of menstruation. The study was approved by the institutional review board at the University of Massachusetts, Amherst. Verbal and written informed consent was obtained from all subjects.

\subsection{Preliminary tests}

Body composition was assessed using dual-energy x-ray absorptiometry (Lunar, Madison, WI). Subjects then performed a continuous progressive exercise test either on a cycle ergometer (SensorMedics 800, Yorba Linda, CA) or a treadmill (Life Fitness 9100HR, Schiller Park, IL) to determine peak oxygen consumption. Briefly, after a 5-minute warm-up, the workload on the cycle ergometer or the grade of the treadmill was increased every 2 minutes until the subject was unable to maintain the required pedal cadence or running speed. During the test, oxygen consumption and carbon dioxide production were assessed using an online metabolic system (Parvomedics TrueMax 2400, Consentius Technologies, Sandy, UT), and $\mathrm{VO}_{2}$ peak was defined as the highest $\mathrm{VO}_{2}$ value obtained (30-second average).

\subsection{Estimated energy expenditure and energy intake}

Before the intervention, all subjects completed a 3-day diet recall to provide an initial estimate of energy intake. Resting energy expenditure (REE) was measured in the morning after an overnight fast using indirect calorimetry to estimate energy requirements. After a 20 -minute period of relaxation, subjects sat comfortably in a reclining chair for 20 minutes while expired air was collected using the metabolic measurement system (Parvomedics TrueMax 2400, Consentius Technologies, Sandy, UT). The measured REE was multiplied by an activity factor from 1.5 to 1.8 dependent upon the subject's individual habitual physical activity (based on physical training logs) to provide an estimate of total energy expenditure. Kien and Ugrasbul [18] reported that energy requirements estimated from the REE and the appropriate activity factor were strongly correlated $(r=0.73)$ with energy requirements measured during 28 days of controlled feeding. Subjects were provided with all meals throughout the study. The standardized diet was composed of $50 \%$ to $60 \%$ carbohydrate, $25 \%$ to $30 \%$ fat, and $15 \%$ to $20 \%$ protein, and consisted of common whole and frozen foods. Subjects were asked to consume discrete meals at certain times of the day (breakfast, lunch, dinner, snacks, etc). Subjects were instructed to consume all food and drinks provided and to return the used containers at the end of each day including any uneaten food. No food was returned, indicating that subjects consumed all foods given. To minimize confounding effects of caffeine and alcohol, subjects were instructed to refrain from both during the intervention.

\subsection{Experimental protocol}

An overview of the study design is shown in Fig. 1. Baseline testing was conducted after 2 days in which subjects performed no structured exercise (ie, no physical activity beyond daily living) and maintained energy balance as described previously. Reduced levels of physical activity were accounted for in the calculated energy requirements. On the morning of day 3 , after a 10 - to 12 -hour overnight fast, a catheter was inserted into a forearm vein, and a fasting blood sample was taken followed by a standard 2-hour oral glucose tolerance test (OGTT). Subjects ingested $75 \mathrm{~g}$ of glucose (Sun Dex, Fisherbrand, Fisher Healthcare, Houston, TX) within 5 minutes, and blood samples were collected every 30 minutes for the next 2 hours. Subjects were overfed by $25 \%$ above their estimated daily energy needs for the next 3 days while continuing to refrain from structured exercise 
Table 1

Energy intake, estimated energy expenditure, and estimated energy balance

\begin{tabular}{cccc}
\hline & Baseline & OF & OF + EX \\
\hline Energy intake, kJ/d & $12088(3117)$ & $15410(3782)$ & $18376(4816)$ \\
CHO, g/d & $399(97)$, & $529(123)$, & $632(158)$, \\
& $55 \%$ & $57 \%$ & $57 \%$ \\
Fat, g/d & $95(37)$, & $120(46)$, & $143(54)$, \\
& $29 \%$ & $28 \%$ & $28 \%$ \\
Pro, g/d & $112(32)$, & $129(32)$, & $150(37)$, \\
& $16 \%$ & $15 \%$ & $15 \%$ \\
Estimated energy & $12171(2979)$ & $12196(2996)$ & $15251(3920)$ \\
expenditure, kJ/d & & & \\
Estimated energy & $-84(456)$ & $+3213(849)$ & $+3125(933)$ \\
balance, kJ/d & & & \\
\hline
\end{tabular}

Values are mean (SD). CHO indicates carbohydrate; Pro, protein.

(OF) to induce a state of relative insulin insensitivity. The composition of the diet was held constant by increasing carbohydrate, protein, and fat intake in a proportional manner (Table 1). After an overnight fast, a second OGTT was given on the morning of day 6 . After the OGTT, subjects performed a single bout of exercise comprising $25 \%$ of their estimated daily energy expenditure (Table 1). Subjects completed the exercise protocol on a cycle ergometer or treadmill at $60 \%$ of $\mathrm{VO}_{2}$ peak until $25 \%$ of baseline energy intake was expended (mean duration, 60 minutes). To ensure that the exercise intensity and energy expenditure were correct, oxygen consumption was measured during the initial 20 minutes of exercise, and heart rate was monitored throughout the protocol. To maintain the desired energy surplus, dietary energy was increased by an additional $50 \%$ to offset the energy expended during exercise (OF and exercise $[\mathrm{OF}+\mathrm{EX}]$; Fig. 1, day 6). As a typical example, a subject consuming $3000 \mathrm{kcal} / \mathrm{d}$ at baseline would have consumed $3750 \mathrm{kcal} / \mathrm{d}$ during OF (energy surplus $=750$ $\mathrm{kcal})$. They would have then consumed $4500 \mathrm{kcal} / \mathrm{d}$ during $\mathrm{OF}+\mathrm{EX}$, which was offset by an exercise expenditure of $750 \mathrm{kcal}$ to maintain an energy surplus of $750 \mathrm{kcal}$. The last OGTT was given the morning after OF + EX.

\subsection{Biochemical analyses}

Venous blood samples were collected in sterile syringes and transferred to tubes containing sodium fluoride (to inhibit glycolysis) and potassium oxalate (for analysis of glucose concentration), EDTA (for analysis of insulin concentration), or a serum separator for analysis of nonesterified fatty acid (NEFA) concentrations. Samples were immediately centrifuged at $3000 \mathrm{~g}$ for 15 minutes, and plasma was aliquoted into polystyrene tubes and stored at $-80^{\circ} \mathrm{C}$ until analyzed. Plasma glucose concentrations were assessed by the glucose oxidase method using an autoanalyzer (Analox Instruments, Lunenburg, MA). Plasma insulin concentrations were determined by radioimmunoassay (Linco Research, St Charles, MO), and plasma NEFA (Wako Chemicals, Richmond, VA) concentrations were determined using an enzymatic colorimetric assay kit.

\subsection{Calculations}

Glucose and insulin concentrations were used to calculate area under the glucose and insulin curves during the OGTT using the trapezoidal method. The values were also used to calculate the composite insulin sensitivity index (C-ISI) [19] and the homeostasis model assessment of insulin resistance (HOMA-IR) [20] as:

$\mathrm{C}-\mathrm{ISI}=\frac{10,000}{\sqrt{(\mathrm{FPG} \times \mathrm{FPI}) \times(G \times I)}}$

$\mathrm{HOMA}-\mathrm{IR}=\frac{(\mathrm{FPG} \times \mathrm{FPI})}{22.5}$

where FPG and FPI are fasting plasma glucose and fasting plasma insulin concentrations, and $G$ and $I$ are the mean glucose and insulin concentrations during the OGTT.

\subsection{Statistical analysis}

A commercial software package from SAS (Cary, NC) was used for statistical analysis of data. For glucose and insulin concentrations during the OGTT, a mixed-model 2-way analysis of variance with repeated measures using a compound symmetric covariate structure was used to test for day $\times$ time interactions. For the glucose and insulin areas under the curve, C-ISI scores, and fasting measurements, a 1-way analysis of variance with a compound symmetric covariate structure was used to test for differences between conditions. In general, data are presented in tables as the condition mean, difference from the baseline condition, the $95 \%$ confidence interval of that difference, and the exact $P$ value. Insulin concentrations were not normally distributed and so the values were $\log$ transformed before statistical analysis. By convention, significant differences were defined as $P<.05$. When appropriate, post hoc tests of significance were performed with a Tukey honestly significant difference test. Although technically complying with the protocol, 1 subject performed an extremely strenuous bout of unaccustomed exercise 3 days before the baseline

Table 2

Fasting plasma glucose, insulin, and NEFAs

\begin{tabular}{lccc}
\hline & Baseline & OF & OF + EX \\
\hline Glucose $(\mathrm{mmol} / \mathrm{L})$ & $5.3(0.7)$ & $5.4(0.6)$ & $5.4(0.6)$ \\
$\Delta$ from baseline & & 0.1 & 0.1 \\
$95 \%$ CI & & $(-0.5,0.3)$ & $(-0.5,0.3)$ \\
Exact $P$ & & 0.463 & 0.653 \\
Insulin $(\mathrm{pmol} / \mathrm{L})$ & $52.8(21.9)$ & $71.9(38.1)$ & $75.7(33.0)$ \\
$\Delta$ from baseline & & 19.1 & 22.9 \\
$95 \%$ CI & & $(-41.2,3.2)$ & $(-45.1,-0.7)$ \\
Exact $P$ & & 0.099 & $0.043^{\mathrm{a}}$ \\
NEFA (mmol/L) & $0.44(0.21)$ & $0.30(0.09)$ & $0.33(0.11)$ \\
$\Delta$ from baseline & & -0.14 & -0.11 \\
95\% CI & & $(0.28,0.02)$ & $(0.24,-0.02)$ \\
Exact $P$ & & $0.024^{\mathrm{a}}$ & 0.108 \\
\hline
\end{tabular}

Values are mean (SD). $\Delta$ indicates group mean difference; $\mathrm{CI}$, confidence interval.

a Significantly different than baseline. 
A
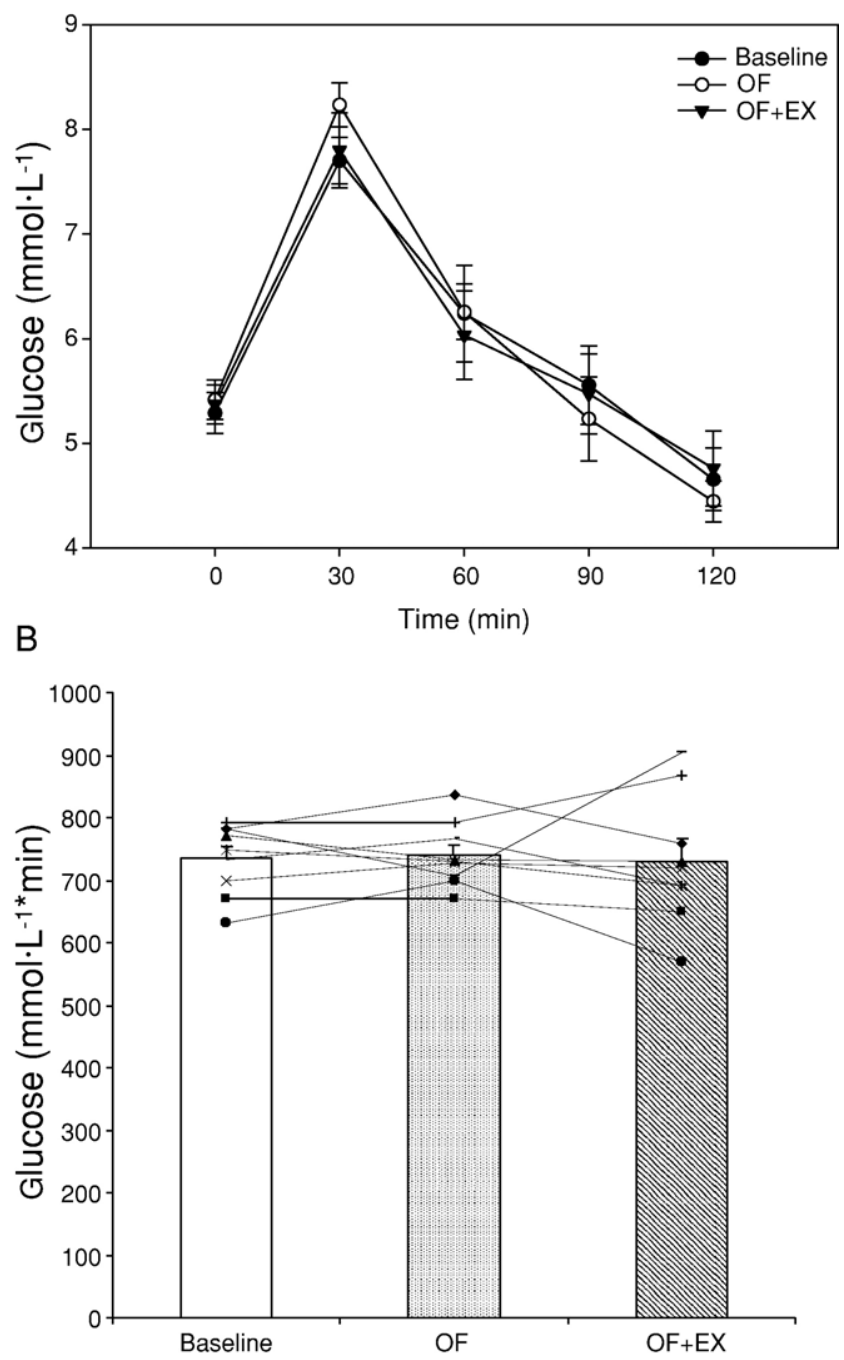

Fig. 2. Plasma glucose response during the 2-hour OGTT (A) and glucose area under the curve with individual data points (B) the morning after each period. Values are mean (SEM).

testing and experienced considerable muscle soreness during the study period. Her data were omitted from the final analysis because the insulin response during the 2-hour OGTT on the OF + EX day was more than 3 SDs from the group mean, as indicated by the auto-regressive integrated moving average model.

\section{Results}

3.1. Energy intake, estimated energy expenditure, and estimated energy balance

By design, energy intake was increased by $25 \%$ for OF and $50 \%$ for OF + EX compared with baseline intake (Table 1). The macronutrient composition of the diet did not change during the intervention because the absolute amounts of ingested carbohydrate, fat, and protein were increased proportionally. Estimated energy expenditure was similar for baseline and OF (Table 1). As designed, exercise energy expenditure $(3063 \pm 803 \mathrm{~kJ})$ increased the estimated energy expenditure during OF + EX. As a result, estimated energy surplus relative to baseline was very similar for $\mathrm{OF}$ and OF + EX (Table 1). Compared with baseline, body mass increased $0.8 \pm 0.6 \mathrm{~kg}$ after OF $(P=.038)$ and a total of $1.1 \pm 0.7 \mathrm{~kg}$ after OF $+\operatorname{EX}(P<.001)$.

\subsection{Effects of the OF period}

Energy surplus without exercise (OF) had no impact on fasting glucose concentration, glucose response during the OGTT, or the glucose AUC (Table 2 and Fig. 2). Mean fasting insulin concentration was $36 \%$ higher ( 7 of 9 subjects showed an increase) after OF, but not significantly different from the baseline (Table 2). Relative to baseline, insulin concentrations during the OGTT were significantly higher at 30 and 60 minutes $(P<.05)$. The insulin AUC was
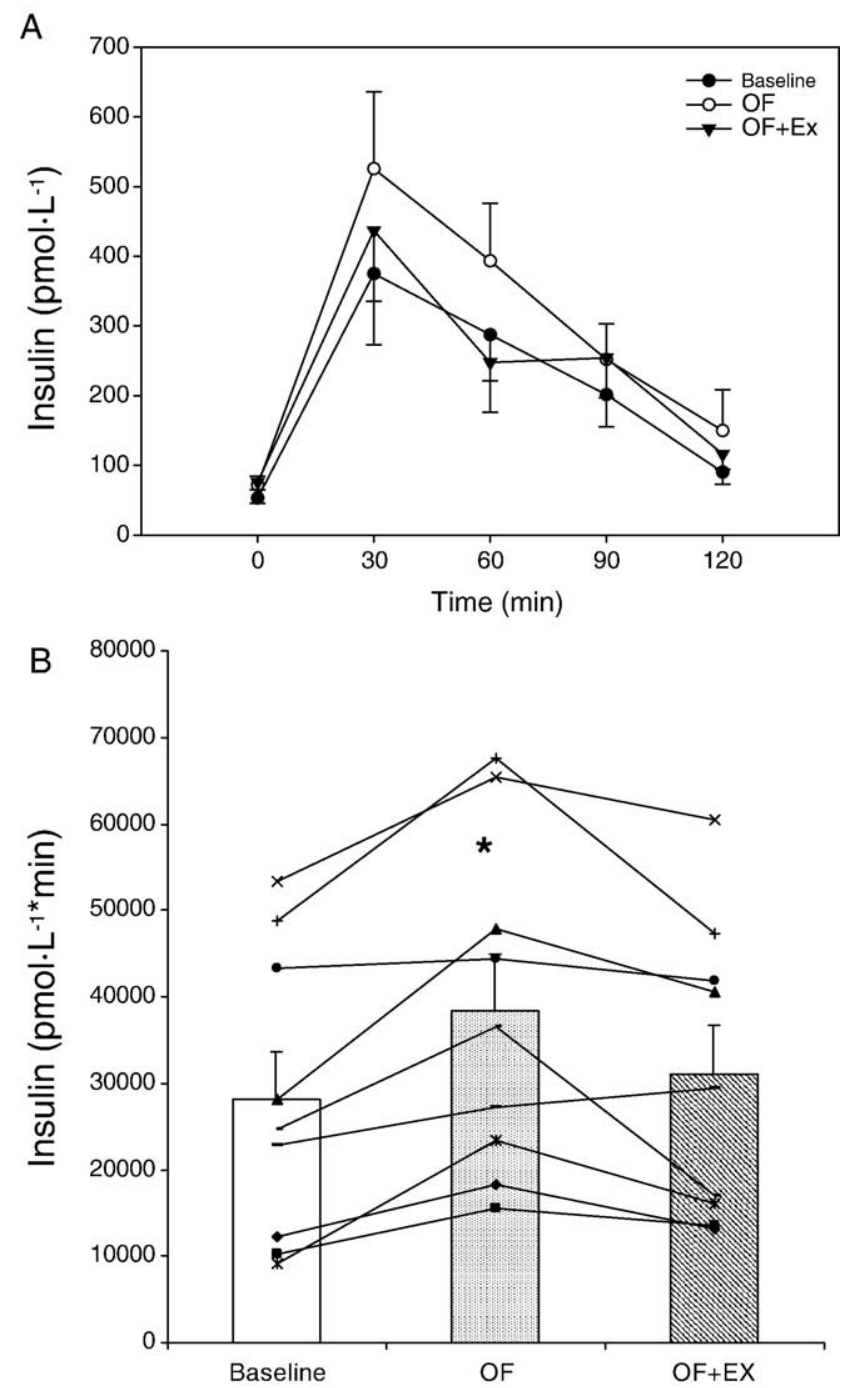

Fig. 3. Plasma insulin response during the 2-hour OGTT (A) and insulin area under the curve with individual data points (B) the morning after each period. Values are mean (SEM). * Significantly different than baseline $(P=.002)$ and $\mathrm{OF}+\mathrm{EX}(P=.048)$. 
significantly elevated by $38 \%$ (all 9 subjects showed an increase) after OF relative to baseline (Fig. 3). Compared with baseline, the C-ISI score was significantly lower (indicating less insulin sensitivity) after OF $(4.6 \pm 1.8$ vs $6.6 \pm 3.1$, respectively; $P=.007)$. The HOMA-IR tended to be higher after OF $(2.9 \pm 1.6)$ than after baseline $(2.1 \pm$ $0.9)$, but the mean difference was not statistically significant despite 7 of the 9 subjects having elevated scores (indicating more insulin resistance) after OF $(P=.123)$. Fasting plasma NEFA concentration was significantly $(-32 \%)$ lower after OF relative to baseline (Table 2).

\subsection{Effects of combined $O F+E X$}

Energy surplus combined with exercise (OF + EX) had no effect on the fasting glucose concentration, the glucose response during the OGTT, or the glucose AUC (Table 2 and Fig. 2). Compared with $\mathrm{OF}$, fasting plasma insulin concentrations remained elevated after $\mathrm{OF}+\mathrm{EX}$ and were significantly $(+43 \%)$ higher than baseline values (Table 2 ). Compared with OF, insulin concentrations during the OGTT were lower after $\mathrm{OF}+\mathrm{EX}(P<.05)$ with no difference between baseline concentrations (Fig. 3). Relative to OF, the mean insulin AUC was significantly lower $(-20 \%)$ after OF + EX, and this effect was consistent as insulin AUC was reduced toward baseline in 8 of the 9 subjects. However, the insulin AUC was reduced to the pre-OF baseline in only 2 of 9 subjects and remained 11\% higher (but not significantly different) than baseline. Relative to OF, the C-ISI score was not significantly increased by $\mathrm{OF}+\mathrm{EX}$ $(5.2 \pm 2.3)$ and remained lower compared with baseline $(6.6 \pm 3.1)$ in all 9 subjects $(P=.058)$. The HOMA-IR remained approximately the same after OF + EX compared with OF $(3.0 \pm 1.4$ and $2.9 \pm 1.6$, respectively). Fasting plasma NEFAs were similar (difference between means of only $7 \%$ ) after OF + EX relative to OF (Table 2).

\section{Discussion}

The goal of the current investigation was to assess the combined impact of exercise and energy surplus on estimated insulin action. Specifically, we assessed whether a single exercise bout would reverse the insulin insensitivity caused by short-term OF and detraining, even in the context of an ongoing energy surplus. The main findings were that (1) as anticipated, 3 days of energy surplus combined with detraining induced a state of insulin insensitivity in healthy individuals as indicated by increases in fasting plasma insulin concentrations, the insulin response to glucose ingestion, and mathematical indices of insulin resistance; (2) with continued energy surplus, a single bout of exercise lowered, but did not completely reverse, the elevated insulin response to glucose that was induced by OF and detraining; and (3) other measures of insulin resistance, for example, C-ISI, fasting insulin concentration, and HOMA-IR, were not affected by the single exercise bout combined with energy surplus.
Short-term energy balance clearly modulates insulin sensitivity. Energy deficit, with or without exercise, improves insulin sensitivity $[5,21,22]$, whereas short-term energy surplus (3-7 days) usually leads to a relative insulin resistance [13-15]. For example, Bachmann et al [13] demonstrated that 3 days OF $(+767 \mathrm{kcal} / \mathrm{d}$ compared with a control group) decreased insulin sensitivity in healthy men. These investigations suggest that OF-induced changes in insulin sensitivity occur in a few days, well before appreciable weight gain. We took advantage of this metabolic response to create a "model" of mild insulin resistance in a population of healthy insulin-sensitive individuals. Similar to prior studies [13-15], we observed a sharp increase in the insulin response to glucose and a significant reduction in the insulin sensitivity index with only a very modest weight gain $(<1 \mathrm{~kg})$. The effect may be attributable to contributions from the considerable energy surplus ( $25 \%$ over estimated requirements) and a reduction in habitual physical activity (ie, detraining). To minimize the potential confounding impact of detraining, we enforced 2 sedentary days before the baseline measures were taken. It is likely, however, that the effects of detraining [4] continued during the 3 days of OF and may have added to the impact of energy surplus alone. Although the continued detraining confounds attribution of the metabolic changes to an independent effect of energy surplus, the main purpose of the energy surplus period was to create a state of mild insulin insensitivity, which was used as a new baseline to assess the impact of exercise combined with energy surplus. From that perspective, the independent effects of energy surplus and detraining are not directly germane to the main purpose of the study. If the goal is to tease out the independent effects of energy surplus and detraining, the study design would require using a subject population composed of sedentary men and/or women.

Because the daily energy surplus was maintained, by design, at $\sim 3183 \mathrm{~kJ} / \mathrm{d}$, the cumulative energy surplus on day $7(+1276 \mathrm{~kJ})$ was higher than on day $6(+9623 \mathrm{~kJ})$. The greater total energy surplus may have independently contributed to insulin insensitivity and minimized the impact of the exercise bout to reverse the effects of overfeeding.

Many researchers have shown that insulin action is enhanced for 24 to 72 hours postexercise, at least when exercise energy expenditure is not replaced $[1,2,5,6]$. When energy balance is restored by the appropriate increase in dietary energy, the effect of exercise on insulin action may be abolished $[5,12]$, considerably blunted $[9,10]$, or maintained [8], depending on the composition, timing, and energy content of the replacement energy (as well as other nondietary factors such as duration and intensity of exercise, short-term exercise or long-term training, obese or lean subjects, etc). There are few prior data relevant to a situation in which energy expended is exceeded by replacement of dietary energy to create an energy surplus. Heled et al $[16,17]$ showed that exercise training ( $\geq 4$ weeks) paired with OF increases insulin sensitivity and delays the 
progression of type 2 diabetes mellitus in overfed Psammomys obesus rats, but not to the same extent as an energy balance condition. Recently, Schenk et al [11] reported that, compared with a saline infusion, a lipid infusion to create an energy surplus of $1100 \mathrm{kcal}$ did not alter insulin sensitivity measured the morning after a short-term bout of exercise. We found that the insulin response to glucose ingestion was reduced by about $20 \%$ after the exercise day compared with sedentary OF days, although energy consumption was raised to maintain the net energy surplus of $25 \%$ over energy need. The reduction only reversed about half of the OF-induced insulin resistance (the insulin area was completely restored to pre-OF values in only 2 subjects), however. In this respect, our data are very consistent with those of Heled et al $[16,17]$ and are opposed to the results in humans from Schenk et al [11]. The other measures in this investigation, for example, C-ISI, fasting insulin, and HOMA-IR, showed no reversal toward baseline after OF + EX and are somewhat consistent with Heled et al $[16,17]$.

The composition of the postexercise energy intake may explain some of the inconsistencies in insulin action. Although the study parameters were considerably different, Cartee et al [1] and Garcia-Roves et al [23] have shown that consumption of carbohydrate foods resulting in replenishment of glycogen stores is a potent stimulus to reverse the exercise-induced enhancement of insulin sensitivity in glycogen-depleted rats. In the recent study by Schenk et al [11], the energy intake after the single bout of exercise was infused in the form of pure lipid and contained no carbohydrate. We chose to more closely simulate "realworld" conditions in which food was consumed orally and the dietary energy was increased while maintaining the same macronutrient composition. Using this protocol, subjects consumed more carbohydrate (increased by approximately $25 \%$ relative to OF and $50 \%$ compared with baseline) during the OF + EX period. It is certainly possible that increase in dietary carbohydrate, rather than dietary energy per se, contributed to blunt the magnitude of the exerciseinduced enhancement of insulin sensitivity.

The timing of the postexercise energy (and/or carbohydrate) replacement may also impact the magnitude of the exercise effect on insulin sensitivity. Providing replacement energy, especially carbohydrate-containing energy, in the "critical window" for maximizing glycogen replacement (generally accepted to be within the first 1-2 hours postexercise), is likely to strongly oppose the insulin-sensitizing effects of exercise. Cartee et al [1] showed that consumption of carbohydrate foods after a bout of exercise reversed the exercise-induced enhancement of insulin sensitivity in glycogen-depleted rats. Recently, we found that replacing the energy expended during exercise to maintain energy balance totally eliminated the effect of short-term exercise training (6 days) to enhance insulin action in overweight, insulin-resistant subjects [5]. In Black et al [5], exercise energy expenditure was replaced immediately postexercise with relatively high-carbohydrate foods. In the current study, energy replacement was delayed by an average of 95 minutes postexercise (range, 90-120 minutes). It is conceivable that the delay slowed replacement of muscle glycogen and/or regulation of key factors (eg, adenosine monophosphate kinase activity, glycogen synthase, glycogen synthase kinase, etc) that mediate the magnitude and duration of the effects of exercise on insulin sensitivity.

Comparison of our results with those from studies in which more direct measures of insulin-mediated glucose uptake were made (eg, Black et al [5], Ross et al $[9,10]$, Schenk et al [11], Segal et al [12]) is problematic. The OGTT has some advantages: glucose is delivered via the gastrointestinal system, makes a first pass through hepatic tissues, and the dynamic changes in blood glucose and insulin concentrations are more representative of a postprandial state, but insulin sensitivity is not directly measured. Plasma insulin concentrations during an OGTT may only explain approximately one third of the variance in insulin sensitivity [24]. When a mathematical model is used [19] to estimate insulin sensitivity (C-ISI) from glucose and insulin responses to an OGTT, however, there is a very good correlation with insulin sensitivity measured by hyperinsulinemic clamp $(r=0.836)$. We found that the C-ISI was significantly reduced after 3 days of energy surplus (which matched the pattern observed in the insulin response to glucose ingestion). However, unlike the return toward baseline values observed in the insulin response, the C-ISI scores changed very little when continued OF was opposed by a short-term bout of exercise. Similarly, measures made in the fasting state, for example, plasma insulin concentration and HOMA-IR, which indicated less insulin sensitivity after energy surplus alone, showed no reversal toward baseline in response to OF plus exercise. These results suggest that the metabolic response to $\mathrm{OF}+$ EX varies with the type of assessment made (insulin area vs $\mathrm{C}$-ISI) and possibly the physiological state (fasted or insulin-stimulated). Similarly, Heled et al [16,17], who showed that exercise training increased muscle insulin responsiveness even coupled with energy surplus, reported that fasting hyperinsulinemia was not attenuated by exercise training when compared with a group of sedentary rats in similar energy surplus. In a slightly different model, in which insulin resistance was induced in rats by high-fat feeding rather than energy surplus, exercise enhanced insulin-stimulated glucose uptake, but not fasting insulin resistance [25]. It is also possible that the relatively small sample size and modest effect size provided inadequate statistical power to reach statistical significance in some of the metabolic measures (eg, C-ISI after OF + EX, HOMA-IR after OF).

Although insulin resistance would be expected to raise circulating fatty acid concentrations, we found that plasma free fatty acid concentrations in the fasting state were lower after OF and OF + EX compared with baseline. Although clearly preliminary, one potential explanation is a decoupling in the impact of the short-term energy surplus 
model on carbohydrate vs fat metabolism. It is also possible that the protocol is a better model for hyperinsulinemia than overt insulin resistance, at least with respect to the antilipolytic action of insulin. Future work using a more sensitive and quantitative method should be used to assess tissue-specific (muscle, liver, adipocytes, beta cell) effects of energy surplus, with and without exercise, on insulin sensitivity.

In summary, we showed that short-term energy surplus combined with detraining induces a state of relative insulin insensitivity in healthy individuals, and despite an ongoing energy surplus, an acute bout of exercise reduces the insulin response to a glucose challenge. However, the exercise bout, when opposed by energy surplus, had no significant impact on the composite insulin sensitivity index or fasting hyperinsulinemia. More systematic studies exploring the interactions between exercise energy expenditure, meal composition, and meal timing are needed to gain better insight into this area. Optimizing the energy content, macronutrient composition, and timing of meals that follow bouts of exercise may be an extremely important consideration for individuals using physical activity to enhance their metabolic health.

\section{Acknowledgment}

This investigation was supported by a grant from the Glass Family Trust and a Junior Faculty Award (BB) from the American Diabetes Association.

We would like to thank all the volunteers for their time and participation in this investigation. We are grateful to Steve Black, PhD, Carrie Sharoff, MS, Laura Gerson, MS, Brooke Stephens, MS, Rebecca Hasson, MS, Elizabeth Mitchell, and Stuart Chipkin, MD, for helping with various aspects of the study.

\section{References}

[1] Cartee GD, Young DA, Sleeper MD, Zierath J, Wallberg-Henriksson $\mathrm{H}$, Holloszy JO. Prolonged increase in insulin-stimulated glucose transport in muscle after exercise. Am J Physiol 1989;256:E494-9.

[2] Devlin JT, Horton ES. Effects of prior high-intensity exercise on glucose metabolism in normal and insulin-resistant men. Diabetes 1985;34:973-9.

[3] Hayashi T, Wojtaszewski JFP, Goodyear LJ. Exercise regulation of glucose transport in skeletal muscle. Am J Physiol Endocrinol Metab 1997;36:E1039-51.

[4] Heath GW, Gavin III JR, Hinderliter JM, Hagberg JM, Bloomfield SA, Holloszy JO. Effects of exercise and lack of exercise on glucose tolerance and insulin sensitivity. J Appl Physiol 1983;55:512-7.

[5] Black SE, Mitchell E, Freedson PS, Chipkin SR, Braun B. Improved insulin action following short-term exercise training: role of energy and carbohydrate balance. J Appl Physiol 2005.

[6] Braun B, Zimmermann MB, Kretchmer N. Effects of exercise intensity on insulin sensitivity in women with non-insulin-dependent diabetes mellitus. J Appl Physiol 1995;78:300-6.
[7] Duncan GE, Perri MG, Theriaque DW, Hutson AD, Eckel RH, Stacpoole PW. Exercise training, without weight loss, increases insulin sensitivity and postheparin plasma lipase activity in previously sedentary adults. Diabetes Care 2003;26:557-62.

[8] Fox AK, Kaufman AE, Horowitz JF. Adding fat calories to meals after exercise does not alter glucose tolerance. J Appl Physiol 2004;97:11-6.

[9] Ross R, Dagnone D, Jones PJ, et al. Reduction in obesity and related comorbid conditions after diet-induced weight loss or exerciseinduced weight loss in men. A randomized, controlled trial. Ann Intern Med 2000;133:92-103.

[10] Ross R, Janssen I, Dawson J, et al. Exercise-induced reduction in obesity and insulin resistance in women: a randomized controlled trial. Obes Res 2004;12:789-98.

[11] Schenk S, Cook JN, Kaufman AE, Horowitz JF. Postexercise insulin sensitivity is not impaired after an overnight lipid infusion. Am J Physiol Endocrinol Metab 2005;288:E519-25.

[12] Segal KR, Edano A, Abalos A, et al. Effect of exercise training on insulin sensitivity and glucose metabolism in lean, obese, and diabetic men. J Appl Physiol 1991;71:2402-11.

[13] Bachmann OP, Dahl DB, Brechtel K, et al. Effects of intravenous and dietary lipid challenge on intramyocellular lipid content and the relation with insulin sensitivity in humans. Diabetes 2001;50: 2579-84

[14] Olefsky J, Crapo PA, Ginsberg H, Reaven GM. Metabolic effects of increased caloric intake in man. Metabolism 1975;24:495-503.

[15] Wang J, Obici S, Morgan K, Barzilai N, Feng Z, Rossetti L. Overfeeding rapidly induces leptin and insulin resistance. Diabetes 2001;50:2786-91.

[16] Heled Y, Shapiro Y, Shani Y, et al. Physical exercise enhances protein kinase $\mathrm{C}$ activity and insulin receptor tyrosine phosphorylation in diabetes-prone Psammomys obesus. Metabolism 2003;52:1028-33.

[17] Heled Y, Shapiro Y, Shani Y, et al. Physical exercise prevents the development of type 2 diabetes mellitus in Psammomys obesus. Am J Physiol Endocrinol Metab 2002;282:E370-5.

[18] Kien CL, Ugrasbul F. Prediction of daily energy expenditure during a feeding trial using measurements of resting energy expenditure, fatfree mass, or Harris-Benedict equations. Am J Clin Nutr 2004;80: 876-80.

[19] Matsuda M, DeFronzo RA. Insulin sensitivity indices obtained from oral glucose tolerance testing: comparison with the euglycemic insulin clamp. Diabetes Care 1999;22:1462-70.

[20] Matthews DR, Hosker JP, Rudenski AS, Naylor BA, Treachor DF, Turner RC. Homeostasis model assessment: insulin resistance and Bcell function from fasting plasma glucose and insulin concentrations in man. Diabetologia 1985;28:412-9.

[21] Arciero PJ, Vukovich MD, Holloszy JO, Racette SB, Kohrt WM. Comparison of short-term diet and exercise on insulin action in individuals with abnormal glucose tolerance. J Appl Physiol 1999;86: $1930-5$.

[22] Assali AR, Ganor A, Beigel Y, Shafer Z, Hershcovici T, Fainaru M. Insulin resistance in obesity: body-weight or energy balance? J Endocrinol 2001;171:293-8.

[23] Garcia-Roves PM, Han DH, Song Z, Jones TE, Hucker KA, Holloszy JO. Prevention of glycogen supercompensation prolongs the increase in muscle GLUT4 after exercise. Am J Physiol Endocrinol Metab 2003;285:E729-36.

[24] Hollenbeck CB, Chen N, Chen YD, Reaven GM. Relationship between the plasma insulin response to oral glucose and insulinstimulated glucose utilization in normal subjects. Diabetes 1984;33 $460-3$.

[25] Kraegen EW, Storlien LH, Jenkins AB, James DE. Chronic exercise compensates for insulin resistance induced by a high-fat diet in rats. Am J Physiol Endocrinol Metab 1989;19:E242-9. 